\title{
Elk-3 Contributes to the Progression of Liver Fibrosis by Regulating the Epithelial-Mesenchymal Transition
}

\author{
Tian Zhu Li ${ }^{1,2}$, Sung Min Kim ${ }^{1}$, Wonhee Hur ${ }^{1}$, Jung Eun Choi ${ }^{1}$, Jung-Hee Kim ${ }^{1}$, Sung Woo Hong ${ }^{1}$, Eun Byul Lee ${ }^{1}$, Joon Ho \\ Lee $^{1}$, and Seung Kew Yoon ${ }^{1}$ \\ ${ }^{1}$ The Catholic University Liver Research Center \& WHO Collaborating Center of Viral Hepatitis, The Catholic University of Korea College of \\ Medicine, Seoul, Korea, and ${ }^{2}$ Molecular Medicine Research Center, Chifeng University School of Medical Science, Chifeng, China
}

\section{See editorial on page 11.}

Background/Aims: The role of Elk-3 in the epithelial-mesenchymal transition (EMT) during liver fibrogenesis remains unclear. Here, we determined the expression of Elk-3 in in vitro and in vivo models and in human liver fibrotic tissues. We also investigated the molecular relationships among Elk3, early growth response-1 (Egr-1), and the mitogen activated protein kinases (MAPK) pathway during EMT in hepatocytes. Methods: We established an in vitro EMT model in which normal mouse hepatocyte cell lines were treated with transforming growth factor (TGF)- $\beta 1$ and a $\mathrm{CCl}_{4}$-induced liver fibrosis model. Characteristics of EMT were determined by evaluating the expression levels of related markers. The expression of Elk-3 and its target Egr-1 were analyzed using Western blotting. Gene silencing of Elk-3 was performed using an siRNA knockdown system. Results: The expression levels of mesenchymal markers were increased during TGF- $\beta 1$-induced EMT of hepatocytes. The expression levels of Elk-3 and Egr-1 were significantly $(p<0.05)$ increased during the EMT of hepatocytes, in $\mathrm{CCl}_{4}$-induced mouse liver fibrotic tissues, and in human liver cirrhotic tissues. Silencing of Elk-3 and inhibition of the Ras-Elk-3 pathway with an inhibitor suppressed the expression of EMT-related markers. Moreover, Elk-3 expression was regulated by $\mathrm{p} 38$ MAPK phosphorylation during EMT. Conclusions: Elk-3 contributes to the progression of liver fibrosis by modulating the EMT via the regulation of Egr1 under MAPK signaling. (Gut Liver 2017;11:102-111)

Key Words: Epithelial-mesenchymal transition; Elk-3; Liver cirrhosis; Early growth response-1; MAPK pathway

\section{INTRODUCTION}

Hepatic fibrosis is caused by excessive accumulation of scar tissue in response to chronic liver injury due to infection, alcohol, chemicals, metabolic disorder, and immune attack. ${ }^{1}$ These wound healing responses, also called fibrogenesis can persist and progress unless adequate treatments are provided. They can ultimately lead to serious end-stage liver diseases through orchestrated signaling networks that regulate the deposition of extracellular matrix (ECM). Subsequently, the excessive accumulation of ECM can result in the destruction of hepatic structure and liver dysfunction, leading to chronic liver failure.

Hepatic injury by various stimuli can activate stellate cells by growth factors, cytokines, and chemokines produced by recruited inflammatory cells. Activated stellate cells can become myofibroblasts, which in turn can produce ECM by autocrine stimulation, leading to tissue contraction due to contractile myofibroblasts. ${ }^{1}$ Although the origin of fibrogenic cells in fibrotic liver is still under debate, quiescent hepatic stellate cells are generally considered to be the main source of fibrogenic cells. $^{2-4}$

Recently, new emerging concept has suggested that epithelial-mesenchymal transition (EMT) could contribute to hepatic fibrogenesis. EMT refers a dynamic and multistep process in which the phenotypic features of epithelial cells are lost. However, they can acquire the properties of mesenchymal cells such as fibroblasts. ${ }^{5}$ Recent study has demonstrated that hepatocytes can contribute to the accumulation of activated fibroblasts via EMT. ${ }^{6}$ In addition, EMT plays an important role in wound healing and cancer metastasis as well as organ fibrosis. ${ }^{7}$ Among many cytokines involved in the process of EMT, transforming growth factor (TGF)- $\beta 1$ plays a key role in the initiation of

Correspondence to: Seung Kew Yoon

Department of Internal Medicine, Seoul St. Mary’s Hospital, 222 Banpo-daero, Seocho-gu, Seoul 06591, Korea

Tel: +82-2-2258-7534, Fax: +82-2-536-9559, E-mail: yoonsk@catholic.ac.kr

Received on November 4, 2015. Revised on January 23, 2016. Accepted on March 22, 2016. Published online August 19, 2016 pISSN 1976-2283 eISSN 2005-1212 https://doi.org/10.5009/gnl15566

(a) This is an Open Access article distributed under the terms of the Creative Commons Attribution Non-Commercial License (http://creativecommons.org/licenses/by-nc/4.0) which permits unrestricted non-commercial use, distribution, and reproduction in any medium, provided the original work is properly cited. 
EMT. $^{8}$ Recent studies have provided evidence that TGF- $\beta 1$ can initiate EMT of hepatocytes, leading to hepatic fibrosis. ${ }^{6,9}$

Three ternary complex factors (TCFs) comprising Elk-1, Sap-1, and Elk-3 (Net/Sap-2/Erp) form a subfamily of Ets-domain transcription factors. ${ }^{10}$ They are involved in the development, cell transformation, and tumor generation. ${ }^{11}$ In particular, Elk-3 plays an important role in wound healing, angiogenesis, cell migration, and tumorigenesis. ${ }^{12}$ Several studies have documented that growth factor and Ras oncogene can induce mitogen activated protein kinase (MAPK) signaling and that its phosphorylation can enhance TCFs, leading to transcriptional activation of several immediate early response genes. ${ }^{13}$ Likewise, Elk-3 is activated by the expression of Ras ${ }^{14}$ and phosphorylated by ERK and p38. ${ }^{15}$ However, the role of Elk-3 in the process of EMT induced by TGF- $\beta 1$ in liver fibrosis remains unclear. Moreover, the biochemical link between Elk-3 and MAPK during hepatic fibrogenesis is still poorly understood.

Early growth response-1 (Egr-1), a zinc finger-containing transcription factor, is immediately expressed in response to a variety of stimuli, such as growth factors and lipopolysaccharide. ${ }^{16,17}$ It has been reported that Egr-1 can regulate genes involved in wound-healing process ${ }^{18}$ and immune response. ${ }^{19}$ Although little is currently known about the function of Egr1 in the process of EMT, a recent study showed that the fibrotic response was profoundly worsened in Egr-1-deficient mice, ${ }^{20}$ suggesting that it is a negative regulator of hepatic fibrosis. Based on previous results, we investigated the underlying molecular mechanisms involved in the cross talk between Elk-3 and MAPK signaling. We also determined whether Elk-3 regulates Egr-1 in TGF- $\beta 1$-induced EMT model.

\section{MATERIALS AND METHODS}

\section{Mice}

C57BL/6 and Balb/c mice were purchased from Orient Bio (Seongnam, Korea) and housed in a standard laboratory animal facility. All animal care and experimental protocols were in accordance with the guidelines for the Care and Use of Laboratory Animals provided by the Research Supporting Center for Medical Science of the Catholic University of Korea.

\section{Carbon tetrachloride induced liver fibrosis model}

Liver fibrosis in Balb/c mice was induced by intraperitoneal injection of carbon tetrachloride $\left(\mathrm{CCl}_{4}\right.$; Sigma, St. Louis, MO, USA) as previously described.6 Briefly, 6-week-old Balb/c mice were injected with $\mathrm{CCl}_{4}$ (1:1 olive oil, $250 \mu \mathrm{L}$ of $20 \% \mathrm{v} / \mathrm{v} \mathrm{CCl}_{4}$ ) intraperitoneally twice a week for 8 weeks to induce liver fibrosis.

\section{Human liver specimens with chronic liver diseases}

Human liver samples liver tissues stored in liquid nitrogen. They were obtained from liver biopsy of patients with chronic liver diseases prior to 2010. All patients provided written in- formed consents for storage of liver tissue samples according to the ethical guidelines of Seoul St. Mary's Hospital in the Catholic University of Korea. Their personal information was restricted for analysis purpose. Such information is not available to the public.

\section{Isolation and primary culture of mouse hepatocyte, and induction of EMT in vitro}

C57BL/6 mice were intraperitoneally anesthetized with Rompun $(10 \mathrm{mg} / \mathrm{kg}$ ) and Zoletil (40 mg/kg). These mice were then exsanguinated. Livers were perfused in situ through portal vein with calcium- and magnesium-free Hanks' balanced salt solution (Welgene, Daegu, Korea) until the firm texture was lost. After perfusion, soft liver was removed and placed in a 1:1 mixture of Dulbecco's modified Eagle's medium and Ham's F-12 medium (DMEM/F12 medium; Invitrogen, Carlsbad, CA, USA). Subsequently, liver suspension was poured through sterile 70- $\mu \mathrm{m}$ nylon mesh (BD Sciences, San Jose, CA, USA) and then homogenate was centrifuged at $50 \mathrm{~g}$ for 2 minutes. Pellet containing parenchymal cells was washed twice with DMEM/F12 medium containing 10\% fetal bovine serum (FBS; Invitrogen). Isolated primary hepatocytes were plated onto collagen coated plates and cultured in DMEM/F12 medium supplemented with 10\% FBS. After attachment, cells were washed with phosphatebuffered saline (PBS) and incubated with serum-free DMEM/ F12 medium containing $5 \mathrm{ng} / \mathrm{mL}$ recombinant human TGF- $\beta 1$ (TGF- $\beta 1$; R \& D Systems, Minneapolis, MN, USA). After 48 hours of seeding, cells without TGF- $\beta 1$ (control) and those with TGF- $\beta 1$ were harvested and further analyzed.

\section{Induction of EMT in vitro with TGF- $\beta 1$ in mouse hepatocyte cell lines}

Nontumorigenic mouse hepatocyte cell lines FL83B (CRL2390) and AML12 (CRL-2254) were purchased from ATCC (Manassas, VA, USA). FL83B cells and AML12 cells were cultured in Ham's F-12K medium (Invitrogen) and DMEM/F12 medium (Gibco, Gaithersburg, MD, USA), respectively. Cells were maintained in a humidified $37^{\circ} \mathrm{C}$ incubator supplied with $5 \%$ $\mathrm{CO}_{2}$.

For in vitro induction of EMT, FL83B cells were incubated with medium containing insulin-transferrin-selenium (ITS; Gibco) for 48 hours prior to TGF- $\beta 1$ treatment. After preincubation, cells were washed three times with PBS and treated with medium containing $3 \mathrm{ng} / \mathrm{mL}$ TGF- $\beta 1$, ITS, and 0.5\% FBS for 48 hours. AML12 cells were washed three times with PBS and treated with serum-free medium containing $1 \mathrm{ng} / \mathrm{mL}$ TGF- $\beta 1$ and ITS for 48 hours. Cells without TGF- $\beta 1$ (control) and those with TGF- $\beta 1$ were harvested and further analyzed.

\section{Small interference RNA transfection}

To investigate the role of Elk-3 during EMT process of hepatocytes, specific small interference RNA (siRNA) targeting Elk- 
3 (siElk-3) and negative control siRNA (siCtrl) were purchased from Santa Cruz Biotechnology (Santa Cruz, CA, USA). These siCtrl and siElk-3 were transfected into FL83B cells and AML12 cells using Lipofectamine ${ }^{\mathrm{TM}} 2000$ transfection reagent (Invitrogen). At 48 hours post transfection, both cells were harvested and subjected to Western blot to determine whether Elk-3 affected EMT induced by TGF- $\beta 1$.

\section{Effect of Ras-Elk-3 pathway inhibitor and p38 MAPK inhibitor on TGF- $\beta 1$ induced EMT}

Recent study has revealed that TGF- $\beta 1$ could activate ERK and p38 MAPK $^{21}$ as well as Elk-3 in hepatocytes. ${ }^{22}$ To investigate whether Elk-3 affected ERK and p38 MAPK signaling pathway during EMT, we treated both control and TGF- $\beta 1$-treated cells with Ras-Elk-3 pathway inhibitor (XRP44X; EMD Millipore Corp., Billerica, MA, USA) and p38 MAPK inhibitor (SB203580; Calbiochem, Nottingham, UK). Briefly, cells were seeded at 60\% confluent in 100-mm culture dishes. They were then treated with $100 \mathrm{nM}$ XRP44X or $10 \mu \mathrm{M}$ SB203580 with or without TGF- $\beta 1$ for 48 hours.

\section{Western blot analysis}

Cells were washed with PBS and lysed in Pro-prep (iNtRon Biotechnology, Houston, TX, USA) containing protease inhibitors on ice for 20 minutes. Protein concentration was determined with Bradford assay (Bio-Rad Laboratories, Hercules, CA, USA). Extracted proteins were separated by $10 \%$ sodium dodecyl sulfate-polyacrylamide gel electrophoresis (SDS-PAGE) and transferred to nitrocellulose membranes (Whatman, Maidstone, UK). After blocking with 5\% skim milk for 30 minutes, membranes were incubated with monoclonal mouse anti- $\beta$ actin (Sigma), monoclonal rabbit anti-E-cadherin (Cell Signaling Technology, Beverly, MA, USA), monoclonal mouse anti$\beta$-catenin (BD Sciences), monoclonal rabbit anti-vimentin (Cell Signaling Technology), monoclonal mouse anti- $\alpha$-SMA (Sigma), polyclonal rabbit anti-Elk-3 (Abcam, Cambridge, MA, USA), monoclonal mouse anti-Egr-1 (Cell Signaling Technology), polyclonal rabbit anti-ERK (Santa Cruz Biotechnology), monoclonal rabbit anti-pERK (Cell Signaling Technology), monoclonal rabbit anti-p38/MAPK (Cell Signaling Technology), and polyclonal rabbit anti-p-p38 MAPK (Cell Signaling Technology) at $4^{\circ} \mathrm{C}$ overnight. Each membrane was washed three times with Tris buffered saline (TBS) containing 0.05\% Tween 20. After incubating with 1:5,000 dilution of horseradish peroxidase conjugated anti-mouse or anti-rabbit immunoglobulin G (IgG; Santa Cruz Biotechnology) for 1 hour. Membranes were washed three times with TBS containing 0.05\% Tween 20. Target protein bands were visualized with an enhanced chemiluminescence system (Amersham Biosciences, Piscataway, NJ, USA) according to the manufacturer's instructions.

\section{Statistics analysis}

All experiments were performed with at least three independent replicates. Statistical analysis was carried out using software Sigma Plot 2000 (Systat Software Inc., San Jose, CA, USA). Student t-test was used for comparison. Statistical significance was considered when p-value was less than 0.05 .

\section{RESULTS}

\section{Expression of Elk-3 in $\mathrm{CCl}_{4}$-induced mouse liver fibrosis model and human liver tissues with chronic liver diseases}

To investigate whether Elk-3 expression was associated with liver fibrosis, we established $\mathrm{CCl}_{4}$-induced mouse liver fibrosis model. The expression levels of $\alpha$-smooth muscle actin ( $\alpha$-SMA, a marker of fibroblast activation) in fibrotic livers were significantly $(\mathrm{p}<0.05)$ higher than those in control livers (Fig. 1A). To investigate whether hepatocytes underwent EMT in $\mathrm{CCl}_{4}$ induced mouse liver fibrosis model, the expressions levels of EMT markers E-cadherin and vimentin were determined by Western blot. The expression levels of E-cadherin protein was significantly $(\mathrm{p}<0.05)$ decreased while that of vimentin was significantly $(\mathrm{p}<0.05)$ increased in fibrotic livers at 8 weeks after $\mathrm{CCl}_{4}$ treatment (Fig. 1A), suggesting that hepatocytes acquired fibroblast-like phenotype in mouse models of $\mathrm{CCl}_{4}$-induced liver fibrosis. In addition, the expression of $\alpha$-SMA was significantly $(p<0.05)$ increased in fibrotic mouse livers, suggesting that EMT contributed to liver fibrosis. The expressions level of Elk-3 was significantly $(\mathrm{p}<0.05)$ increased in fibrotic livers at 8 weeks after $\mathrm{CCl}_{4}$ treatment compared to control livers, suggesting that Elk3 contributed to the development of liver fibrosis. To further confirm the contribution of Elk-3 to liver fibrosis, we evaluated the expression of Elk-3 in human liver tissues, including histologically proven chronic hepatitis tissues $(n=7)$ and liver cirrhosis tissues $(n=7)$. The expression levels of E-cadherin in liver cirrhotic tissues were significantly $(p<0.05)$ lower than those in chronic hepatitis tissues (Fig. 1B). However, the expression levels of Elk-3 in liver cirrhosis tissues were significantly $(\mathrm{p}<0.05)$ higher than those in chronic hepatitis tissues. Taken together, these results indicated that Elk-3 expression contributed to hepatic fibrosis.

\section{Establishment of EMT in vitro model and evaluation of Elk-3 expression}

In this study, we established EMT in vitro model by treating with TGF- $\beta 1$ in primary hepatocytes isolated from normal adult mice as described by the Zeisberg et al. ${ }^{6}$ The shape of TGF- $\beta 1$ treated mouse primary hepatocytes was changed from round to spindle (Fig. 2A, upper panel). However, no change in shape was observed for those without TGF- $\beta 1$ treatment, suggesting that mature hepatocytes could acquire mesenchymal phenotype. To further validate EMT process in primary hepatocytes, we per- 


\section{A}

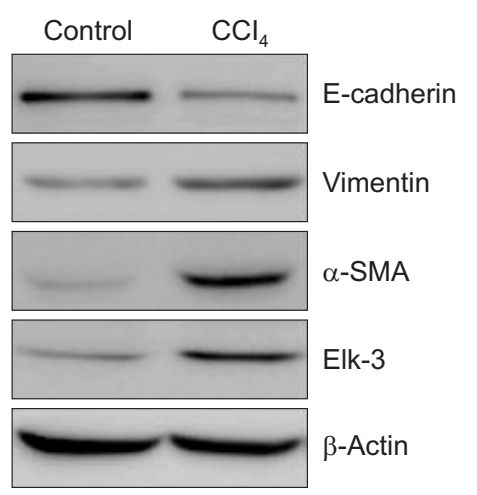

B
E-cadherin
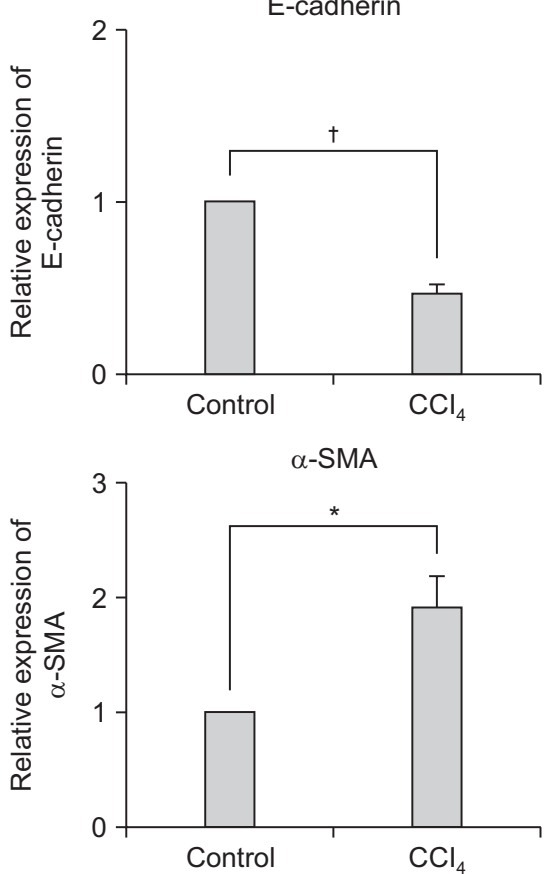
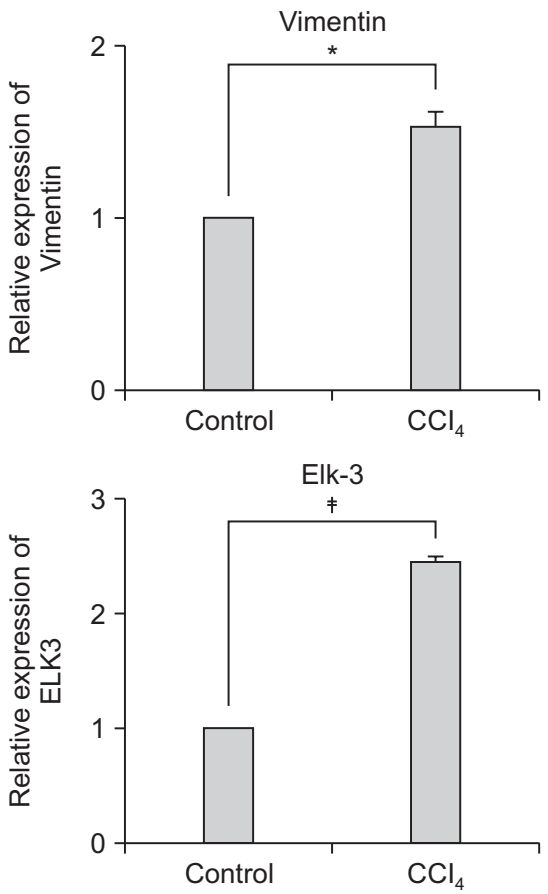

\begin{tabular}{|c|c|c|c|c|c|c|c|c|c|c|c|c|c|c|}
\hline \multirow[b]{2}{*}{ Control } & \multicolumn{7}{|c|}{$\mathrm{CHB}$} & \multicolumn{7}{|c|}{ LC } \\
\hline & 1 & 2 & 3 & 4 & 5 & 6 & 7 & 1 & 2 & 3 & 4 & 5 & 6 & 7 \\
\hline
\end{tabular}

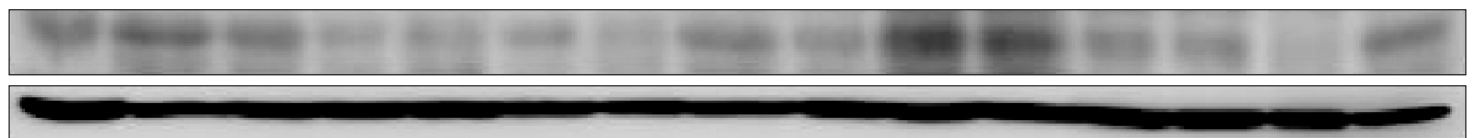

Elk-3

$\beta$-Actin
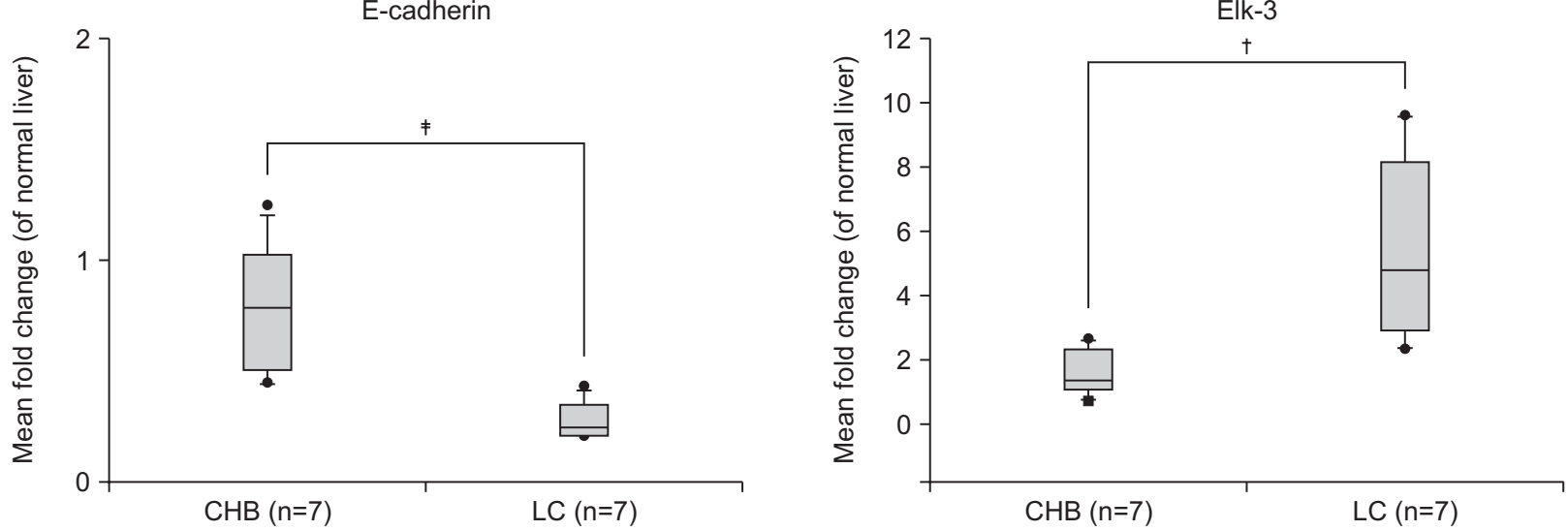

Fig. 1. Upregulation of Elk-3 in mouse and human fibrotic liver tissues. To determine whether Elk-3 is associated with liver fibrogenesis, Elk-3 expression was assessed in mouse (A) and human (B) fibrotic liver tissues. (A) Balb/c mice were intraperitoneally injected with $\mathrm{CCl}_{4}$ twice a week for 8 weeks. They were then sacrificed, and the livers were harvested. The expression levels of E-cadherin, vimentin, $\alpha$-smooth muscle actin ( $\alpha$-SMA), and Elk-3 in $\mathrm{CCl}_{4}$-induced mouse liver tissues were determined by Western blot analysis. (B) Chronic hepatitis B and cirrhosis patient liver tissues were obtained from patients. The expression levels of E-cadherin and Elk-3 in chronic hepatitis $B(n=7)$ and cirrhosis liver tissues ( $n=7)$ were determined using Western blot analysis. The relative expression in the bar graphs was normalized against the expression of $\beta$-actin (reference). The data are presented as the means \pm standard error. All data are representative of at least three independent experiments.

Control, human normal liver lysate; CHB, chronic hepatitis B patient liver tissue lysates; LC, liver cirrhosis patient liver tissue lysates. ${ }^{*} \mathrm{p}<0.05$, ${ }^{\dagger} \mathrm{p}<0.01,{ }^{\ddagger} \mathrm{p}<0.001$. 
A
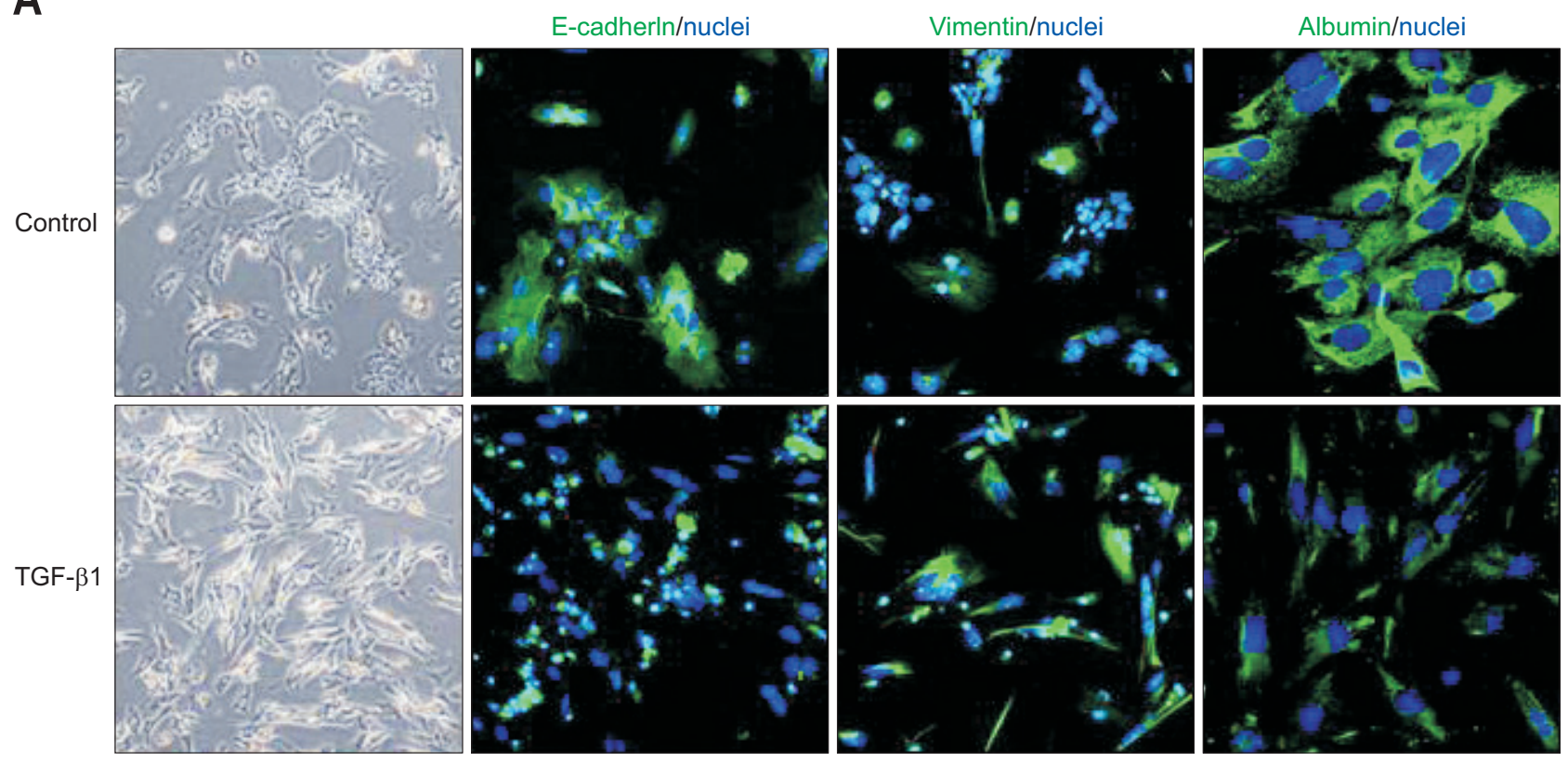

B

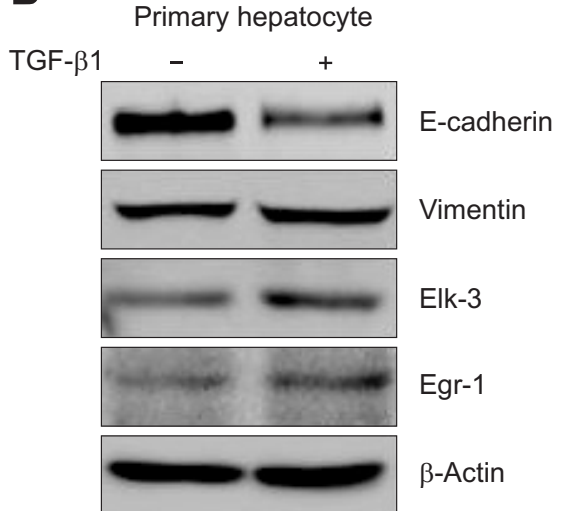

C

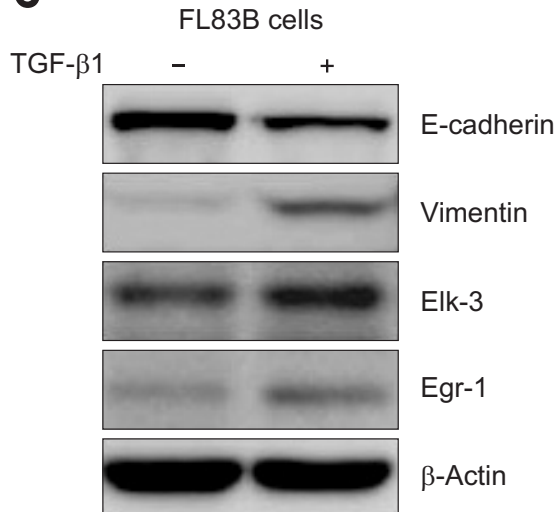

D
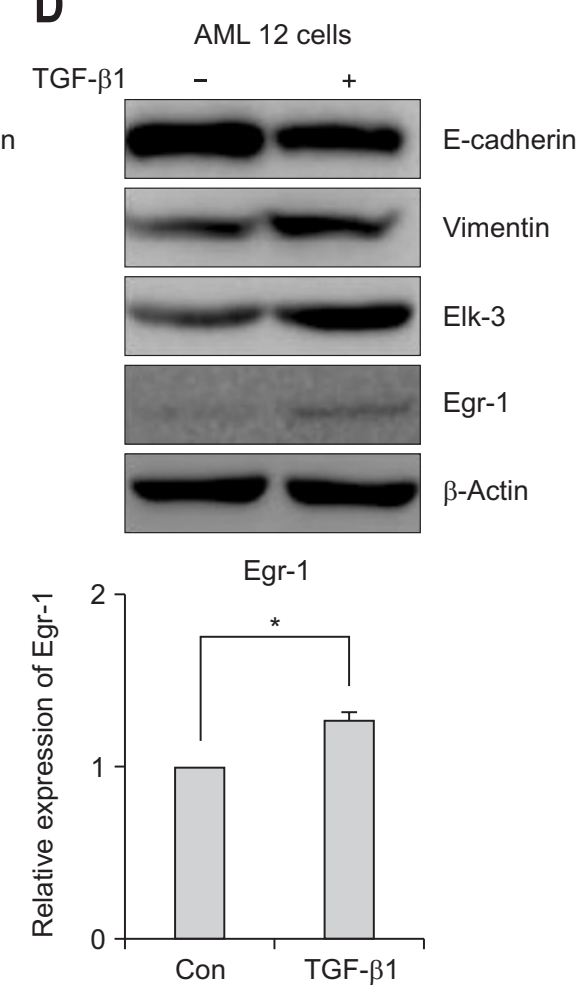
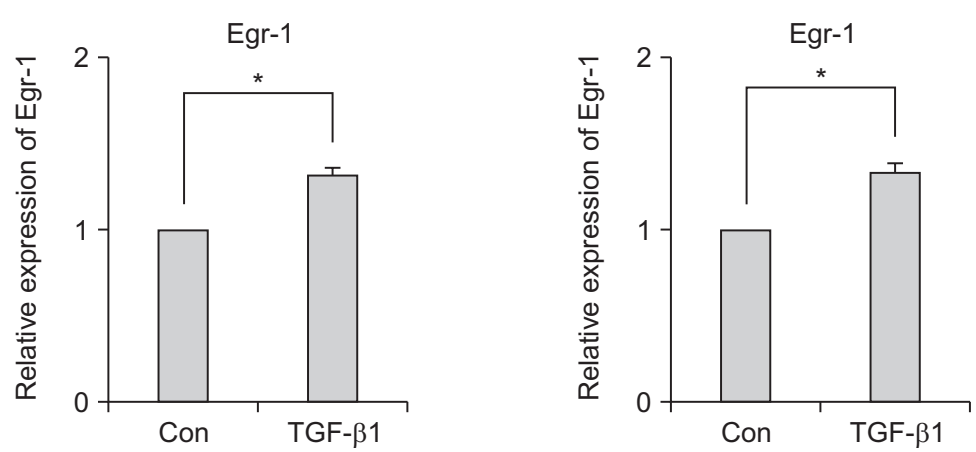

Fig. 2. Expression of the epithelial-mesenchymal transition (EMT) marker genes Elk-3 and early growth response-1 (Egr-1) in hepatocytes after transforming growth factor (TGF)- $\beta 1$ treatment. (A) Primary hepatocytes were treated with $5 \mathrm{ng} / \mathrm{mL}$ TGF- $\beta 1$ to establish the in vitro EMT model. Green, anti-E-cadherin, antivimentin, and antialbumin; blue, DAPI (4',6-diamidino-2-phenylindole). Original magnification $\times 200$. Control, TGF- $\beta 1$ untreated cells; TGF- $\beta 1$, TGF- $\beta$ 1-treated cells. (B-D) We evaluated the EMT features (E-cadherin and vimentin) and Elk-3 and Egr-1 expression in TGF $\beta$-1-treated primary hepatocytes (B), FL83B cells (C), and AML12 cells (D). Relative expression levels in the graphs were normalized against the expression of $\beta$-actin (reference). Values are presented as the means \pm standard error. All data are representative of at least three independent experiments.

Con, TGF- $\beta 1$ untreated cells; TGF- $\beta 1$, TGF- $\beta 1$-treated cells. ${ }^{*} \mathrm{p}<0.05$.

formed immunofluorescent staining with albumin, E-cadherin, and vimentin at 48 hours after TGF- $\beta 1$ treatment. The expression of albumin was decreased over time with fibroblast-like morphological change, indicating that mature hepatocyte could acquire mesenchyme phenotype after TGF- $\beta 1$ treatment (Fig. 2A). In addition, the expression of EMT markers, E-cadherin, 
and vimentin was investigated by western blotting. After treatment with TGF- $\beta 1$ for 48 hours, the expression of E-cadherin was significantly $(\mathrm{p}<0.05)$ reduced in TGF- $\beta 1$ treated primary hepatocytes compared to that in cells without TGF- $\beta 1$ treatment (Fig. 2B). On the other hand, the expression of mesenchymal marker vimentin was significantly $(\mathrm{p}<0.05)$ enhanced in TGF- $\beta 1$ treated primary hepatocytes compared to that in cells without TGF- $\beta 1$ treatment. During the induction of EMT in this model, the expression of ELK3 was significantly $(\mathrm{p}<0.05)$ increased in TGF- $\beta 1$ treated primary compared to cells without TGF- $\beta 1$ treatment (Fig. 2B). Two normal mouse hepatocyte cell lines FL83B and AML12 also showed similar results (Fig. 2C and D). These results provided evidence that TGF- $\beta 1$ induced EMT in mouse hepatocytes, resulting in the loss of their epithelial cellspecific phenotypes but the acquirement of mesenchymal cell features. In addition, the expression of Elk-3 was more prominently observed in EMT hepatocytes induced by TGF- $\beta 1$. Taken together, these results suggested that Elk-3 expression played an important role during EMT process in in vitro setting of liver fibrosis.
A

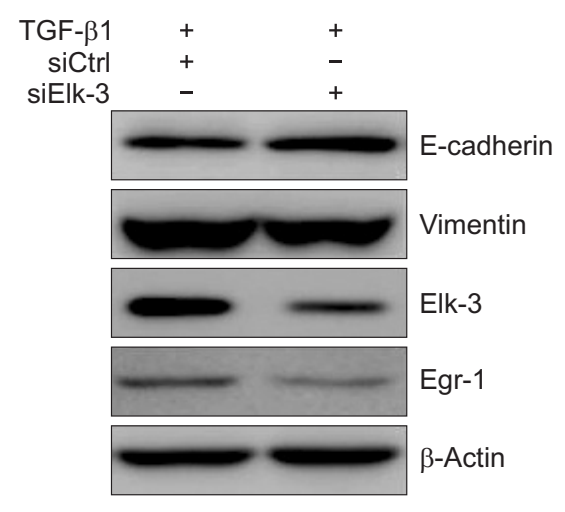

C

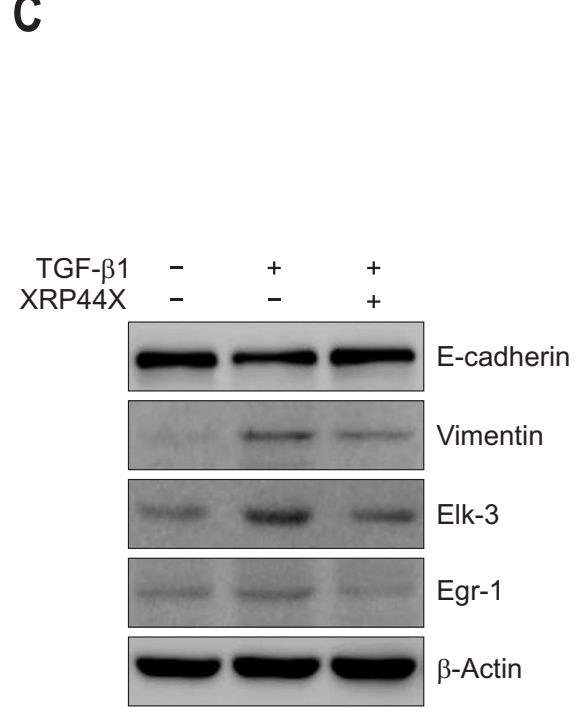

B

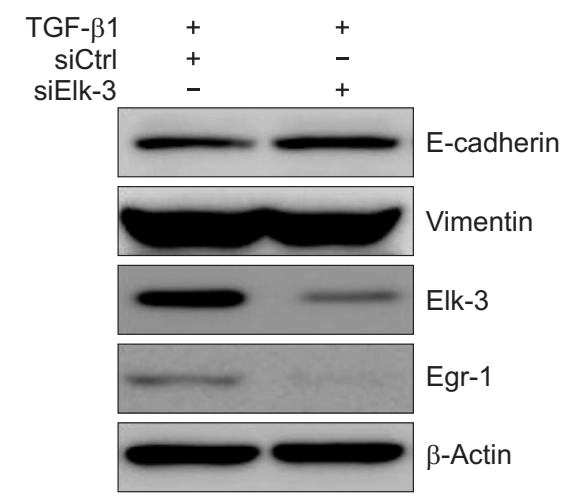

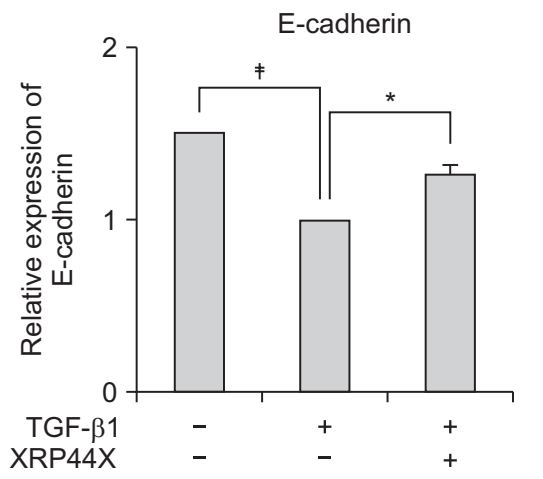

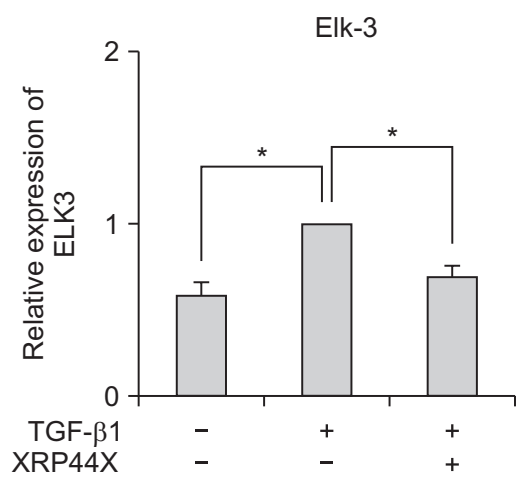

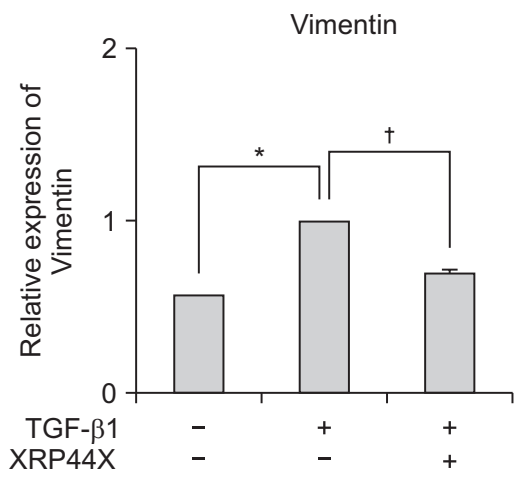

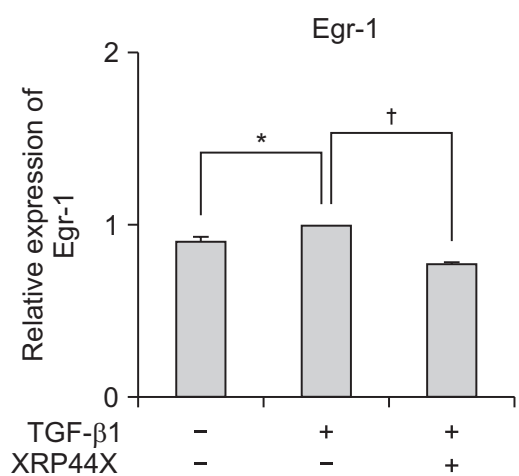

Fig. 3. Inhibition of Elk-3 interrupts transforming growth factor (TGF)- $\beta 1$-induced epithelial-mesenchymal transition (EMT) progression. Elk-3 siRNA at $20 \mathrm{nM}$ was transfected into TGF- $\beta$ 1-treated FL83B cells (A) or AML12 cells (B). At 48 hours posttransfection, the cells were harvested and lysed. Western blot analysis was performed to determine the expression of E-cadherin, vimentin, Elk-3 and early growth response-1 (Egr-1). (C) After treatment with $100 \mathrm{nM}$ of the Ras-Elk-3 pathway inhibitor XRP44X, the expression levels of E-cadherin, vimentin, Elk-3 and Egr-1 were determined. Expression levels were normalized against the expression of $\beta$-actin (reference). Values are presented as the means \pm standard error. All data are representative of at least three independent experiments. ${ }^{*} \mathrm{p}<0.05,{ }^{\dagger} \mathrm{p}<0.01,{ }^{\ddagger} \mathrm{p}<0.001$. 


\section{Egr-1 expression is regulated by Elk-3 expression in TGF- $\beta 1$ induced EMT model}

Recent studies have demonstrated that Egr-1 is a target gene of Elk-3 induced by tissue injury. ${ }^{20,23}$ However, little is known the relationship between Egr-1 and Elk-3 expression during EMT process of liver fibrosis. Thus we investigated whether Egr-1 expression was regulated by Elk- 3 expression in TGF- $\beta 1$ induced EMT model. The expressions levels of Egr-1 and Elk-3 in three cell lines (primary hepatocytes, FL83B cells and AML12 cells) after treatment of TGF- $\beta 1$ were determined. As a result, the expression of Egr-1 was significantly $(\mathrm{p}<0.05)$ higher than that in cells without TGF- $\beta 1$ treatment (Fig. $2 \mathrm{~B}-\mathrm{D}$ ).

In addition, the expression level of Egr-1 was positively correlated with the expression of Elk-3, suggesting that Egr-1 expression was possibly regulated by Elk- 3 in TGF- $\beta 1$ induced EMT hepatocytes. To further define these findings, we silenced Elk-3 expression in FL83B and AML12 cells treated with TGF- $\beta 1$, and then determined the expressions of EMT markers and Egr-1 using Western blot. In TGF- $\beta 1$-induced EMT hepatocytes, the expression level of Egr-1 was downregulated under the suppression of Elk-3 in both cell lines treated with Elk-3 siRNA compared to that in the control (Fig. 3A and B). Furthermore, silencing of Elk-3 resulted in increased expression of Ecadherin but decreased expression of vimentin indicating that Elk-3 silencing could reverse EMT in the process of liver fibrosis.

To further confirm our findings, Ras-Elk-3 pathway inhibitor (XRP44X) was used to treat TGF- $\beta 1$-treated FL83B cells. As shown in Fig. 3C, downregulation of Elk-3 by XRP44X in TGF- $\beta 1$-treated FL83B cells suppressed the Egr-1 expression. Moreover, downregulation of Egr-1 in TGF- $\beta 1$-induced EMT hepatocytes enhanced the expression of E-cadherin and reduced the expression of vimentin, as suggesting that Elk-3 pathway inhibitor might be able to reverse liver fibrosis. Collectively, these results suggest that Elk-3 in conjunction with Egr-1, plays an important role in EMT process and that suppression of Elk-3 might be a promising strategy to reverse liver fibrosis.

\section{Elk-3 contributes TGF- $\beta 1$ induced EMT process via p38 MAPK-Elk-3 pathway}

In order to clarify the signaling pathway involved in TGF- $\beta 1$ induced EMT hepatocyte, we evaluated the expressions levels of ERK and p38 MAPK in TGF- $\beta 1$ treated FL83B cells. As shown in Fig. 4A, no significant difference in phosphorylation of ERK was found between TGF- $\beta 1$ treated FL83B cells and those without TGF- $\beta 1$ treatment. However, the expression of phosphorylated p38 MAPK in TGF- $\beta 1$ treated FL83B cells was significantly $(p<0.05)$ increased compared to that in non-treated FL83B cells. These results suggested that p38 MAPK pathway instead of, ERK pathway was mainly involved in EMT process of hepatocytes.

To determine the possible relationship between p38 MAPK activation and Elk-3 expression, we analyzed the changes of
Elk-3 expression after treating TGF- $\beta 1$ treated FL83B cell s with p38 inhibitor, SB202190. As shown in Fig. 4B, inhibition of P38 MAPK phosphorylation reduced the expression of Elk-3 and Egr-1 with reciprocal mesenchymal-to-epithelial transition. These results provided evidence that Elk-3 and p38 MAPK pathway were involved in TGF- $\beta 1$ induced EMT process in hepatocytes. Taken together, these results demonstrated that Elk3 contributed to TGF- $\beta 1$ induced EMT process via p38 MAPKElk-3 pathway.

\section{DISCUSSION}

Many studies have revealed that EMT plays an important role in the pathogenesis of organ fibrosis ${ }^{24}$ and tumor metastasis. ${ }^{25}$ Since the role of EMT in the process of fibrosis was first demonstrated in the kidney, ${ }^{24}$ a number of studies have been conducted to clarify the mechanism of EMT in pathological settings of organ fibrosis. Recent in vitro and in vivo studies have revealed that EMT plays a pivotal role during hepatic fibrogenesis. ${ }^{6,26}$

In this study, our in vitro data supported the concept that TGF- $\beta 1$ treatment could induce EMT in which mature hepatocytes could acquire mesenchymal phenotype. Therefore, we speculated that these EMT process might contribute to liver fibrogenesis.

It has been demonstrated that wound healing and vascularization in wounded skin of Elk-3 mutant mice are retarded compared to those in wild type mice, ${ }^{27}$ suggesting that Elk3 can regulate angiogenesis in the process of wound healing. However, the role of Elk-3 in hepatic fibrosis in animal model or human is currently unknown. In this study, we revealed for the first time that Elk-3 played a crucial role in the process of liver fibrosis. The expression of Elk-3 in fibrotic livers of the mice treated with $\mathrm{CCl}_{4}$ for 8 weeks was significantly higher than that of control mice (Fig. 1A). Similar findings were observed in human liver tissues. The expression of Elk-3 in liver tissues of patients with liver cirrhosis expression was also significantly higher than that in liver tissues of patients with those with chronic hepatitis (Fig. 1B). Moreover, the expression of Elk-3 was inversely correlated with the expression of E-cadherin in mouse fibrotic liver and human cirrhotic liver tissues. Furthermore, silencing of Elk-3 resulted in increased expression of Ecadherin but decreased expression of vimentin, indicating that Elk-3 silencing could reverse EMT in the process of liver fibrosis. Taken together, these results provide compelling evidence that Elk-3 plays a critical role in hepatic fibrogenesis. However, how Elk-3 contributes to the process of hepatic fibrosis remains unclear. Elk-3 is a transcriptional repressor under basal conditions. It is known target of Ras pathway. ${ }^{12}$

In this study, we also investigated the expression of Egr-1, a known target gene of Elk-3, in hepatocyte EMT in vitro model and evaluated the relationship between the expressions of Egr-1 and Elk-3. In TGF- $\beta 1$-induced EMT hepatocytes, the expression 
A

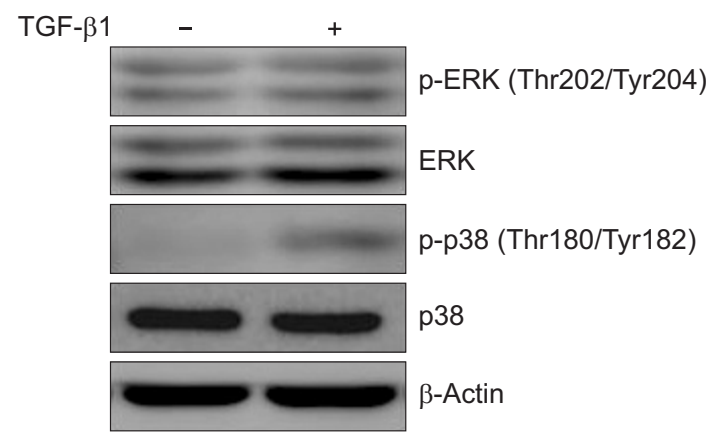

B
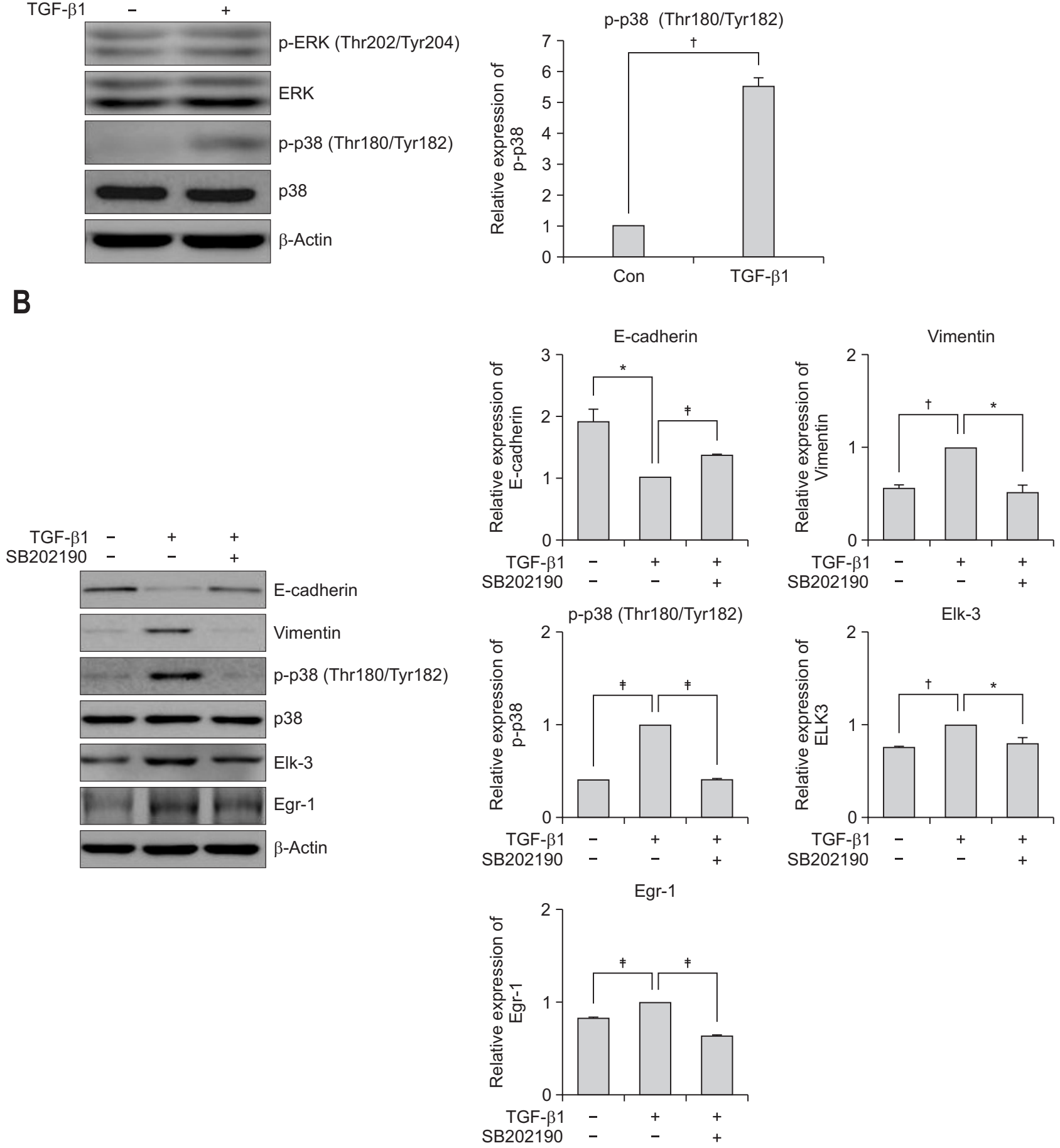

Fig. 4. Suppression of Elk-3 function prevents p38/MAPK-induced epithelial-mesenchymal transition (EMT) progression after transforming growth factor (TGF)- $\beta 1$ treatment. (A) Expression of ERK and p38/MAPK in TGF- $\beta 1$-treated FL83B cells. Bands show the active and inactive form of ERK and p38/MAPK expression after TGF- $\beta 1$ treatment. (B) Protein expression levels of E-cadherin, vimentin, p-p38, p38, Elk-3 and Egr-1 detected in $3 \mathrm{ng} / \mathrm{mL}$ TGF- $\beta 1$ and $10 \mu \mathrm{M}$ p38/MAPK inhibitor (SB203580)-treated FL83B cells for 48 hours. Protein expression was detected by Western blot analysis. Expression levels were normalized against the expression of $\beta$-actin (reference). Values are presented as the means \pm standard error. All data are representative of at least three independent experiments. MAPK, mitogen activated protein kinase; Egr-1, early growth response-1. ${ }^{*} \mathrm{p}<0.05,{ }^{\dagger} \mathrm{p}<0.01,{ }^{\ddagger} \mathrm{p}<0.001$.

of Egr-1 was significantly upregulated. In addition, the upregulation of Egr-1 was significantly correlated with higher expression of Elk-3. Furthermore, Egr-1 expression was downregulated under the suppression of Elk-3 in mouse hepatocyte cells treated with Elk-3 siRNA. These results suggested that Egr-1 regulated by Elk-3 contributed to the EMT process of hepatocyte during 
hepatic fibrogenesis. Wu et $a{ }^{28}{ }^{28}$ has reported that the healing process in cutaneous wound of mice harboring null mutation of Egr-1 is retarded, reducing the influx of inflammatory cells in the dermis. In addition, the expression of Egr-1 is enhanced in fibrotic tissue of mice with bleomycin-induced scleroderma. ${ }^{29}$ Importantly, Egr-1 has been shown to participate in mediating TGF- $\beta$ signaling, which is known to affect metastatic behavior of cancer cells. ${ }^{30,31}$ Many studies have shown that the target genes of Egr-1 in mesenchymal cells are ID1, ID2, Collagen Type I Alpha 2 (COL1A2), and platelet-derived growth factor alpha chain. ${ }^{30,32-38}$ These Egr-1 target genes are already known to play a role in liver fibrogenesis.

Previous in vitro study has demonstrated that Elk-3 is phosphorylated and activated by Ras-Erk signaling pathway, resulting in wound healing and angiogenesis. ${ }^{39}$ They identified a novel pyrazole XRP44X inhibiting fibroblast growth factor 2-induced Elk-3 phosphorylation by the Ras-Erk signaling upstream from Ras. ${ }^{39}$ To further confirm that Elk-3 could regulate Egr-1, we used XRP44X to treat mouse hepatocyte treated with TGF- $\beta 1$. As shown in Fig. 3C, Egr-1 was downregulated by Elk3 via Ras signaling pathway. In addition, the downregulation of Elk-3 in TGF- $\beta 1$-induced EMT hepatocytes enhanced the expression of E-cadherin but reduced the expression of vimentin suggesting that Elk-3 inhibitor might be able to reverse liver fibrosis. In consistent with previous results, our study demonstrated that Elk-3 in EMT hepatocytes induced by TGF- $\beta 1$ might drive fibrotic response through regulation of Egr-1. To better understand the network of signaling involved in EMT induced by TGF- $\beta 1$, we determined the expression levels of ERK and p38 MAPK in TGF- $\beta 1$ induced EMT hepatocytes. The expression level of phosphorylated p38 MAPK in TGF- $\beta 1$ induced EMT hepatocytes was significantly higher compared to that in the control. However, phosphorylated ERK did not show significant difference compared to the control, suggesting that p38 MAPK pathway instead of ERK was, mainly involved in EMT process of hepatocytes. These results were in consistent with a previous report that TGF- $\beta 1$ could activate p38 MAPK, leading to apoptosis and EMT. ${ }^{40}$ To further validate this finding, treatment with p38 inhibitor SB202190 on TGF- $\beta 1$-induced EMT hepatocyte remarkably diminished the phosphorylation of p38 MAPK, leading to reduced expressions of Elk-3, ultimately resulting in a reciprocal mesenchymal-to-epithelial transition. These results, for the first time, provide evidence that p38 MAPK, Elk-3, and Egr-1 sequentially signaling pathway are involved in TGF- $\beta 1$ induced EMT process in hepatocytes.

In conclusion, our results revealed that hepatocyte EMT triggered by Elk-3 was closely associated with hepatic fibrosis. We also demonstrated that Elk-3 and its target gene Egr-1 played pivot roles in the EMT process, leading to hepatic fibrosis via p38 MAPK pathway. These results provided a novel molecular mechanism of Elk-3 during hepatic fibrogenesis and suggesting that targeting Elk-3/Egr-1 might be used as a novel therapeutic strategy for hepatic fibrosis.

\section{CONFLICTS OF INTEREST}

No potential conflict of interest relevant to this article was reported.

\section{ACKNOWLEDGEMENTS}

This research was supported by Basic Science Research Program through the National Research Foundation of Korea (NRF) funded by the Ministry of Science, ICT \& Future Planning (NRF2012R1A2A1A01001941 \& 2015R1A2A1A15052783).

\section{REFERENCES}

1. Friedman SL. Mechanisms of hepatic fibrogenesis. Gastroenterology 2008;134:1655-1669.

2. Friedman SL, Roll FJ, Boyles J, Bissell DM. Hepatic lipocytes: the principal collagen-producing cells of normal rat liver. Proc Natl Acad Sci U S A 1985;82:8681-8685.

3. Beaussier M, Wendum D, Schiffer E, et al. Prominent contribution of portal mesenchymal cells to liver fibrosis in ischemic and obstructive cholestatic injuries. Lab Invest 2007;87:292-303.

4. Kisseleva T, Uchinami H, Feirt N, et al. Bone marrow-derived fibrocytes participate in pathogenesis of liver fibrosis. J Hepatol 2006;45:429-438

5. Thiery JP. Epithelial-mesenchymal transitions in tumour progression. Nat Rev Cancer 2002;2:442-454.

6. Zeisberg M, Yang C, Martino M, et al. Fibroblasts derive from hepatocytes in liver fibrosis via epithelial to mesenchymal transition. J Biol Chem 2007;282:23337-23347.

7. Kalluri R, Neilson EG. Epithelial-mesenchymal transition and its implications for fibrosis. J Clin Invest 2003;112:1776-1784.

8. Zavadil J, Böttinger EP. TGF-beta and epithelial-to-mesenchymal transitions. Oncogene 2005;24:5764-5774.

9. Nitta T, Kim JS, Mohuczy D, Behrns KE. Murine cirrhosis induces hepatocyte epithelial mesenchymal transition and alterations in survival signaling pathways. Hepatology 2008;48:909-919.

10. Wasylyk B, Hagman J, Gutierrez-Hartmann A. Ets transcription factors: nuclear effectors of the Ras-MAP-kinase signaling pathway. Trends Biochem Sci 1998;23:213-216.

11. Seth A, Ascione R, Fisher RJ, Mavrothalassitis GJ, Bhat NK, Papas TS. The ets gene family. Cell Growth Differ 1992;3:327-334.

12. Ayadi A, Suelves M, Dollé P, Wasylyk B. Net, an Ets ternary complex transcription factor, is expressed in sites of vasculogenesis, angiogenesis, and chondrogenesis during mouse development. Mech Dev 2001;102:205-208.

13. Treisman R. Ternary complex factors: growth factor regulated transcriptional activators. Curr Opin Genet Dev 1994;4:96-101.

14. Giovane A, Pintzas A, Maira SM, Sobieszczuk P, Wasylyk B. Net, a new ets transcription factor that is activated by Ras. Genes Dev 
1994;8:1502-1513.

15. Price MA, Cruzalegui FH, Treisman R. The p38 and ERK MAP kinase pathways cooperate to activate Ternary Complex Factors and c-fos transcription in response to UV light. EMBO J 1996;15:65526563.

16. Gashler A, Sukhatme VP. Early growth response protein 1 (Egr1): prototype of a zinc-finger family of transcription factors. Prog Nucleic Acid Res Mol Biol 1995;50:191-224.

17. Coleman DL, Bartiss AH, Sukhatme VP, Liu J, Rupprecht HD. Lipopolysaccharide induces Egr-1 mRNA and protein in murine peritoneal macrophages. J Immunol 1992;149:3045-3051.

18. Braddock M. The transcription factor Egr-1: a potential drug in wound healing and tissue repair. Ann Med 2001;33:313-318.

19. McMahon SB, Monroe JG. The role of early growth response gene 1 (egr-1) in regulation of the immune response. J Leukoc Biol 1996;60:159-166.

20. Pritchard MT, Nagy LE. Hepatic fibrosis is enhanced and accompanied by robust oval cell activation after chronic carbon tetrachloride administration to Egr-1-deficient mice. Am J Pathol 2010;176:2743-2752.

21. Zhang YE. Non-Smad pathways in TGF-beta signaling. Cell Res 2009;19:128-139.

22. Grotegut S, von Schweinitz D, Christofori G, Lehembre F. Hepatocyte growth factor induces cell scattering through MAPK/Egr1-mediated upregulation of Snail. EMBO J 2006;25:3534-3545.

23. McMullen MR, Pritchard MT, Wang Q, Millward CA, Croniger CM, Nagy LE. Early growth response-1 transcription factor is essential for ethanol-induced fatty liver injury in mice. Gastroenterology 2005;128:2066-2076.

24. Strutz F, Okada H, Lo CW, et al. Identification and characterization of a fibroblast marker: FSP1. J Cell Biol 1995;130:393-405.

25. Thompson EW, Newgreen DF, Tarin D. Carcinoma invasion and metastasis: a role for epithelial-mesenchymal transition? Cancer Res 2005;65:5991-5995.

26. Kaimori A, Potter J, Kaimori JY, Wang C, Mezey E, Koteish A. Transforming growth factor-beta1 induces an epithelial-to-mesenchymal transition state in mouse hepatocytes in vitro. J Biol Chem 2007;282:22089-22101.

27. Zheng H, Wasylyk C, Ayadi A, et al. The transcription factor Net regulates the angiogenic switch. Genes Dev 2003;17:2283-2297.

28. Wu M, Melichian DS, de la Garza M, et al. Essential roles for early growth response transcription factor Egr-1 in tissue fibrosis and wound healing. Am J Pathol 2009;175:1041-1055.

29. Bhattacharyya S, Chen SJ, Wu M, et al. Smad-independent trans- forming growth factor-beta regulation of early growth response-1 and sustained expression in fibrosis: implications for scleroderma. Am J Pathol 2008;173:1085-1099.

30. Chen SJ, Ning H, Ishida W, et al. The early-immediate gene EGR-1 is induced by transforming growth factor-beta and mediates stimulation of collagen gene expression. J Biol Chem 2006;281:2118321197.

31. Bhattacharyya S, Ishida $\mathrm{W}, \mathrm{Wu} \mathrm{M}$, et al. A non-Smad mechanism of fibroblast activation by transforming growth factor-beta via cAbl and Egr-1: selective modulation by imatinib mesylate. Oncogene 2009;28:1285-1297.

32. Tournay 0, Benezra R. Transcription of the dominant-negative helix-loop-helix protein Id 1 is regulated by a protein complex containing the immediate-early response gene Egr-1. Mol Cell Biol 1996;16:2418-2430.

33. Zhu X, Lin Y, Bacanamwo M, et al. Interleukin-1 beta-induced Id2 gene expression is mediated by Egr-1 in vascular smooth muscle cells. Cardiovasc Res 2007;76:141-148.

34. Li W, Cai Z, Liu M, et al. Urotensin II contributes to collagen synthesis and up-regulates Egr-1 expression in cultured pulmonary arterial smooth muscle cells through the ERK1/2 pathway. Biochem Biophys Res Commun 2015;467:1076-1082.

35. Tkalcević VI, Cuzić S, Brajsa K, et al. Enhancement by PL 14736 of granulation and collagen organization in healing wounds and the potential role of egr-1 expression. Eur J Pharmacol 2007;570:212221.

36. Chen J, Xu L, Chen S, Yang J, Jiang H. Transcriptional regulation of platelet-derived growth factor-B chain by thrombin in endothelial cells: involvement of Egr-1 and CREB-binding protein. Mol Cell Biochem 2012;366:81-87.

37. Luo Y, Zhang M, Zhang J, et al. Platelet-derived growth factor induces Rad expression through Egr-1 in vascular smooth muscle cells. PLoS One 2011;6:e19408.

38. Li X, Meng Y, Huang ML, Zhang XL, Zhang ZS. Angiotensin II stimulates platelet-derived growth factor-B expression in hepatic stellate cells by activating EGR-1. Nan Fang Yi Ke Da Xue Xue Bao 2008;28:963-967.

39. Wasylyk C, Zheng H, Castell C, Debussche L, Multon MC, Wasylyk B. Inhibition of the Ras-Net (Elk-3) pathway by a novel pyrazole that affects microtubules. Cancer Res 2008;68:1275-1283.

40. Yu L, Hébert MC, Zhang YE. TGF-beta receptor-activated p38 MAP kinase mediates Smad-independent TGF-beta responses. EMBO J 2002;21:3749-3759. 